\title{
An integrated vendor-buyer model with stock-dependent demand
}

\author{
Mohsen S. Sajadieh ${ }^{\mathrm{a}, *}$, Anders Thorstenson ${ }^{\mathrm{b}}$, Mohammad R. Akbari Jokar ${ }^{\mathrm{a}}$ \\ ${ }^{a}$ Department of Industrial Engineering, Sharif University of Technology, Tehran, Iran \\ ${ }^{b}$ CORAL - Centre for OR Applications in Logistics, Department of Business Studies, \\ Aarhus School of Business, Aarhus University, Denmark
}

\begin{abstract}
We develop an integrated vendor-buyer model for a two-stage supply chain. The vendor manufactures the product and delivers it in a number of equal-sized batches to the buyer. The items delivered are presented to the end customers in a display area. Demand is assumed to be positively dependent on the amount of items displayed. The objective is to maximize total supply chain profit. The numerical analysis shows that buyer-vendor coordination is more profitable in situations when demand is more stock dependent. It also shows that the effect of double marginalization provides a link between the non-coordinated and the coordinated case.
\end{abstract}

Keywords: Batch production; Double marginalization; Inventory; Integrated vendor-buyer model; Stock-dependent demand.

\footnotetext{
* Corresponding author. E-mail: msajadieh@mehr.sharif.edu
} 


\section{Introduction}

In order to satisfy customer demands in today's competitive markets, critical information needs to be shared along the supply chain. A high level of coordination between vendors' and buyers' decision making is also required. The concept of joint economic lot sizing (JELS) has been introduced to refine traditional methods for independent inventory control. The purpose is to find a more profitable joint production and inventory policy, as compared to the policy resulting from independent decision making.

The idea of optimizing the joint total cost in a single-vendor, single-buyer model was considered early on by Goyal (1976). Banerjee (1986) developed the model by incorporating a finite production rate and following a lot-for-lot policy for the vendor. By relaxing Banerjee's lot-for-lot assumption, Goyal (1988) proposed a more general joint economic lotsizing model. Lu (1995) specified the optimal production and shipment policies when the shipment sizes are equal. He relaxed the assumption of Goyal (1988) about completing a whole batch before starting shipments. Goyal (1995) then developed a model where successive shipment sizes increase by a ratio equal to the production rate divided by the demand rate. He found an expression for the optimal first shipment size as a function of the number of shipments. Later, Hill (1997) took this idea one step further by considering the geometric growth factor as a decision variable. He suggested a solution method based on an exhaustive search for both the growth factor and the number of shipments within certain ranges. Finally, Hill (1999) determined the form of the overall optimal policy. This turns out to be a combination of the policy suggested by Goyal (1995) used initially and an equal shipments policy used subsequently. However, because the policy with equal-sized shipments between the vendor and the buyer is straightforward to implement in practice, this shipment policy is usually employed in the JELS modeling literature. 
The basic JELS model has been extended in several different directions. The literature on JELS may thus be divided into different categories treating issues such as quality (e.g., Affisco et al., 2002), controllable lead time (e.g., Hoque and Goyal, 2006), stochastic lead time (e.g., Sajadieh et al., 2009), multiple buyers (e.g., Chan and Kingsman, 2007), setup and order-cost reduction (e.g., Chang et al., 2006), transportation (e.g., Ertogral et al., 2007), deteriorating items (e.g., Yang and Wee, 2000), fuzzy logic (e.g., Pan and Yang, 2008), and three-level supply chains (e.g., Khouja, 2003). Some contributions to the literature may belong to more than one of these categories. Moreover, Hill and Omar (2006) derived the optimal policy of an integrated production-inventory model where, contrary to most of the previous work, it is assumed that the unit stock-holding cost decreases as stock moves downstream in the supply chain. We refer to Ben-Daya et al. (2008) for a comprehensive review of the JELS literature. It is beyond the scope of this paper to discuss all contributions in detail.

Demand in inventory control models is most commonly assumed to be exogenous, although models with partly endogenous demand exist. In the marketing literature there is empirical evidence showing that consumer demand may indeed vary with the inventory on display or on the shelf at a retailer. For example, an investigation by Desmet and Renaudin (1998) supported the hypothesis that direct shelf-space elasticities are significantly non-zero for many product categories. In particular, they concluded that product categories typical of impulse buying have higher space elasticities. Moreover, Koschat (2008) provided empirical evidence from magazine retailing and demonstrated that the demand for a specific brand decreases as the on-shelf inventory of that brand decreases. Gupta and Vrat (1986), and Baker and Urban (1988) were among the first to introduce a class of inventory models in which the demand rate is inventory dependent. They considered a single-period model, where the 
demand is a polynomial function of the inventory on hand. Several other contributions extended this model to other inventory situations. Balakrishnan et al. (2004) and Urban (2005) conducted comprehensive reviews of inventory models for products exhibiting inventory-dependent demand. In particular, the latter studied two types of models in which the demand rate is either a function of the initial inventory level, or it depends on the currently available inventory level. Recently, Hariga et al. (2007) proposed an inventory model to determine the product assortment, inventory replenishment policy, display area, and shelf-space allocation decisions that maximize the retailer's profit. Abbott and Palekar (2008) studied a single-store, multi-product inventory problem in which product sales are a composite function of the shelf space. Warburton (2009) also considered the stock-dependent demand problem.

Wang and Gerchak (2001) developed models for coordinating decentralized two-stage supply chains when demand is shelf-space dependent. They characterized retailers' Nash equilibrium and explored whether the manufacturer can use incentives to coordinate such supply chains. Zhou et al. (2008) also considered the coordination issues in a decentralized two-echelon supply chain, but in cases where the manufacturer follows a lot-for-lot policy, and the demand is dependent on the inventory level on display. The Stackelberg game structure was discussed. Their model provides the manufacturer with a quantity discount scheme to entice the retailer to increase the order quantity. Also recently, Goyal and Chang (2009) proposed an inventory model with both ordering and transfer lot sizes, where the demand rate depends on the stock level displayed. However, they determined the ordering and transfer schedules based on the buyer's costs only.

In this paper, we propose a joint economic lot-sizing model for coordination in a centralized supply chain when determining the optimal vendor and buyer policies. The 
vendor manufactures the product in batches at a finite rate and delivers it in equal-sized transfer lots to the buyer. Some of the delivered items are displayed on the shelves in the buyer's retail store, while the rest of the items are kept in the buyer's warehouse. Final customer demand is positively dependent on the amount of items shown on the shelf/in the display area. The objective is to maximize the total system profit when there is centralized coordination of the supply chain members. In the sense emphasized by the italicized text, our analysis differs from and adds to the contributions referred to in the previous paragraph. The result is then compared to the total profit obtained in the corresponding non-coordinated supply chain. Finally, we also show and discuss how the so-called double marginalization effect (Spengler, 1950; Jeuland and Shugan, 1983; Weng, 1995) impacts the performance of the supply chain in the non-coordinated case. This provides a linkage between the coordinated and the non-coordinated supply chains.

The rest of this paper is organized as follows. In Section 2, the modeling assumptions and notation are provided. In Section 3, we develop the non-coordinated supply chain model and show how to find the independently optimal policies for the buyer and the vendor. We introduce the coordinated supply chain model in Section 4 and develop an algorithm to find the jointly optimal policy. Section 5 uses numerical examples to compare the two models. Conclusions and further research directions are presented in Section 6.

\section{Assumptions and notation}

The following assumptions are used throughout this paper to develop the models proposed:

1. The supply chain consists of one vendor supplying a single product to one buyer, i.e. it forms a bilateral monopoly. 
2. The buyer faces a deterministic consumer demand rate $D(I)$ which is an increasing function of the stock on display $I$. It has the polynomial form (see e.g., Baker and Urban, 1988, Balakrishnan et al., 2004, and Zhou et al., 2008):

$D(I)=\alpha I^{\beta}$, where $\alpha>0$ and $0<\beta<1$ are the scale and the shape parameters, respectively. The shape parameter, $\beta$, reflects the elasticity of the demand rate with respect to the stock level on display.

3. There is a limited capacity $C_{d}$ of the display area, i.e. $I \leq C_{d}$. This limitation could be interpreted as a given shelf space allocated to the product.

4. The vendor has a finite production rate $P$ which is greater than the maximum possible demand rate, i.e. $P>\alpha C_{d}^{\beta}$.

5. At each setup the vendor manufactures a production batch $n_{v} Q$, where $n_{v}$ is an integer and the size of each shipment to the buyer is $Q$.

6. Inventory at the buyer's warehouse is continuously reviewed. The buyer orders a lot of size $Q$ when the inventory level reaches the reorder point. The items are transferred from the warehouse to the display area in $n_{b}$ equal lots of size $q$ until the inventory level in the warehouse falls to zero and a new lot of size $Q$ is delivered. Hence, $Q=n_{b} q$, where $n_{b}$ is an integer.

7. Shortages are not permitted, and transfers to the display area occur as the inventory on display reaches the level zero. This is the so-called run-out replenishment policy for stock-dependent demand models (see Balakrishnan et al., 2004). It is not the optimal policy in general, because some demand may be lost when the inventory on display is low. The run-out replenishment policy is used for simplicity in this multi-stage model.

8. Both the lead time between the vendor and the buyer, and the lead time between the buyer's warehouse and the display area are constant. However, as demand is 
deterministic, we may assume (without loss of generality) that both lead times are zero.

9. Inventory holding costs increase downstream in the supply chain, but they are not related directly to the unit prices.

10. The time horizon is infinite.

A graphical representation of the inventory levels at the vendor, as well as at the two stocking points at the buyer is presented in Figure 1.

\section{Figure 1}

The cost parameters are:

$A_{v} \quad$ Vendor's setup cost

$A_{b} \quad$ Buyer's fixed ordering cost including any fixed delivery cost for each batch shipped to the buyer

$S \quad$ Buyer's fixed cost for transfer from the warehouse to the display area

$c$ The net unit purchasing price (charged by the vendor to the buyer, but net of the vendor's, i.e. the supply chain's, acquisition costs)

$\delta \quad$ The net unit selling price (charged by the buyer to the consumer, but net of the vendor's, i.e. the supply chain's, acquisition costs)

$h_{v} \quad$ Inventory holding cost per unit per unit time at the vendor stage

$h_{w}$ Inventory holding cost per unit per unit time at the buyer's warehouse, $h_{w}>h_{v}$.

$h_{d}$ Inventory holding cost per unit per unit time at the buyer's display area, $h_{d}>h_{w}$.

We note that the simple cost structure suggested here is also in accordance with the theoretical implications for inventory control concluded in Curşeu et al. (2009) from their empirical study of handling operations in grocery retail stores. 


\section{Non-coordinated supply chain}

For comparative purposes, we first obtain the independently optimal policies for the vendor and the buyer, respectively. In this non-coordinated case, each supply chain member tries to maximize its own profit. The result is then compared to the coordinated system (Section 4), where the two parties cooperate and/or are controlled centrally.

\subsection{Buyer's optimal policy}

The objective of the buyer is to maximize its own profit. The elements of the buyer's profit are as follows: the revenue from selling the product, the fixed cost of ordering from the vendor, the holding cost at the warehouse, the holding cost at the display area, the fixed cost of transfer from the warehouse to the display area, and the variable purchasing cost.

The demand rate at time $t$ is equal to the decrease in the inventory level at that time. Therefore, the inventory dynamics $I(t)$ are described by the differential equation

$$
\frac{d I(t)}{d t}=-\alpha I(t)^{\beta}, \quad 0 \leq t \leq T_{d}
$$

where $T_{d}$ is the cycle time defined in Figure 1. Thus, the (on-hand) inventory at time $t$ can be obtained by solving $I(t)^{-\beta} d I(t)=-\alpha d t$. By integrating both sides, we have

$$
\int_{0}^{t} I(t)^{-\beta} d I(t)=-\int_{0}^{t} \alpha d t
$$

Hence,

$$
I(t)^{1-\beta}-I(0)^{1-\beta}=-\alpha(1-\beta) t .
$$

As $I(0)=q$, we get

$$
I(t)=\left[-\alpha(1-\beta) t+q^{1-\beta}\right]^{\frac{1}{1-\beta}} .
$$

Substituting $I\left(T_{d}\right)=0$ into the above expression, we get $T_{d}$ based on the transfer quantity $q$ as $T_{d}=q^{1-\beta} /[\alpha(1-\beta)]$. 
The buyer's total cost $T C_{b w}$ at the warehouse is obtained as

$$
T C_{b w}=\frac{A_{b}}{T_{w}}+\frac{h_{w}}{T_{w}}\left(\frac{n_{b}\left(n_{b}-1\right) q T_{d}}{2}\right)
$$

where $T_{w}$ is the warehouse cycle time specified in Figure 1. Substituting $T_{w}=n_{b} T_{d}$ and $T_{d}=q^{1-}$ ${ }^{\beta} /[\alpha(1-\beta)]$ into the above expression and simplifying, the total cost per unit time at the warehouse is

$$
T C_{b w}=\frac{A_{b} \alpha(1-\beta)}{n_{b} q^{1-\beta}}+\frac{h_{w}\left(n_{b}-1\right) q}{2} .
$$

The buyer's total cost $T C_{b d}$ at the display area consists of the fixed cost of transfer from the warehouse to the display area, and the holding cost at the display area. Therefore,

$$
T C_{b d}=\frac{S}{T_{d}}+\frac{h_{d}}{T_{d}} \int_{0}^{T_{d}} I(t) d_{t}=\frac{S}{T_{d}}+\frac{h_{d}}{T_{d}}\left(\frac{q^{2-\beta}}{\alpha(2-\beta)}\right) .
$$

Substituting $T_{d}=q^{1-\beta} /[\alpha(1-\beta)]$ into the above expression and simplifying, the buyer's total cost per unit time at the display area is

$$
T C_{b d}=\frac{S \alpha(1-\beta)}{q^{1-\beta}}+\frac{h_{d}(1-\beta) q}{2-\beta} .
$$

Total net revenue per unit time is $T R=(\delta-c) q / T_{d}=(\delta-c) \alpha(1-\beta) q^{\beta}$, and the buyer's total profit is $T P_{b}=T R-T C_{b w^{-}} T C_{b d}$. Thus,

$$
T P_{b}\left(q, n_{b}\right)=(\delta-c) \alpha(1-\beta) q^{\beta}-\frac{\alpha(1-\beta)\left(A_{b} / n_{b}+S\right)}{q^{1-\beta}}-\left(\frac{h_{w}\left(n_{b}-1\right)}{2}+\frac{h_{d}(1-\beta)}{2-\beta}\right) q
$$

Taking the second partial derivative of $T_{b}\left(q, n_{b}\right)$ with respect to $q$, we get

$$
\frac{\partial^{2} T P_{b}\left(q, n_{b}\right)}{\partial q^{2}}=-\alpha(1-\beta)^{2} q^{\beta-2}\left[(\delta-c) \beta+(2-\beta)\left(A_{b} / n_{b}+S\right) q^{-1}\right]<0
$$

Hence, $T P_{b}\left(q, n_{b}\right)$ is concave in the transfer quantity $q$ for a given value of $n_{b}$. However, there is no closed-form solution for the optimal $q$. Therefore, we employ a one-dimensional search algorithm to find its optimal value.

Moreover, assume (temporarily) that $n_{b}$ is a continuous variable. Taking the second 
partial derivative of $\operatorname{TP}_{b}\left(q, n_{b}\right)$ with respect to $n_{b}$, we obtain

$$
\frac{\partial^{2} T P_{b}\left(q, n_{b}\right)}{\partial n_{b}^{2}}=-\frac{2 \alpha(1-\beta) A_{b}}{q^{1-\beta} n_{b}^{3}}<0
$$

Thus, $\operatorname{TP}_{b}\left(q, n_{b}\right)$ is also concave in $n_{b}$ for a given value of $q$. Taking the first partial derivative of $T P_{b}\left(q, n_{b}\right)$ with respect to $n_{b}$, and equalizing it to zero, we have

$$
\frac{\partial T P_{b}\left(q, n_{b}\right)}{\partial n_{b}}=\frac{\alpha(1-\beta) A_{b}}{q^{1-\beta} n_{b}^{2}}-\frac{h_{w} q}{2}=0,
$$

from which we obtain $n_{b}^{2}=\frac{2 \alpha(1-\beta) A_{b}}{h_{w} q^{2-\beta}}$.

As expected, there is an inverse relation between $n_{b}$ and $q$ : as the transfer quantity decreases, the number of shipments increases. Although theoretically the transfer quantity is only assumed to be greater than zero, for practical purposes we can assume that it is not less than one. In other words, there is a finite smallest unit for the product which the transfer is based upon. The upper bound for the optimal number of transfers can then be obtained by considering $q=1$ as the smallest possible quantity. Hence,

$$
n_{b}^{\max }=\left\lceil\sqrt{\frac{2 \alpha(1-\beta) A_{b}}{h_{w}}}\right\rceil,
$$

where the upper part brackets indicate rounding up to the nearest integer. The lower bound for the optimal number of transfers can be obtained by considering $q=C_{d}$ as the largest possible transfer quantity. Hence,

$$
n_{b}^{\min }=\max \left\{\left\lfloor\sqrt{\frac{2 \alpha(1-\beta) A_{b}}{h_{w} C_{d}^{2-\beta}}}\right\rfloor, 1\right\},
$$

where the lower part brackets indicate rounding down to the nearest integer. The bounds in (4) and (5) are used in the solution algorithm below for finding the buyer's optimal inventory policy. 
The optimal solution to the buyer's problem can be obtained by using the following algorithmic steps.

Step 1. Initialize by computing $n_{b}^{\max }$ and $n_{b}^{\min }$ using Equations (4) and (5), respectively. Set $n_{b}=n_{b}^{\min }$ and $T P_{b}^{o p t}=0$.

Step 2. Find the value of $q$, employing a one-dimensional search algorithm on Equation (3), so that $T P_{b}\left(q, n_{b}\right)$ is maximized for the given value of $n_{b}$.

Step 3. If $q>C_{d}$, then set $q=C_{d}$, and if $q<1$, then set $q=1$.

Step 4. Compute $T P_{b}\left(q, n_{b}\right)$ using Equation (3).

Step 5. If $T P_{b}\left(q, n_{b}\right)>T P_{b}^{o p t}$, then set $T P_{b}^{o p t}=T P_{b}\left(q, n_{b}\right), n_{b}^{o p t}=n_{b}$, and $q^{o p t}=q$.

Step 6. Increment $n_{b}$ by 1 . If $n_{b} \leq n_{b}^{\max }$, then go to Step 2. Otherwise, the current solution is globally optimal.

Evidently, this algorithm terminates with the optimal solution in a finite number of iterations.

\subsection{Vendor's optimal policy}

When the transfer quantity and the number of transfers have been decided by the buyer, orders are received by the vendor at known intervals $T_{w}$ (see Figure 1). The vendor's average inventory level is then obtained as

$$
\begin{aligned}
\bar{I}_{v} & =\frac{1}{T_{v}}\left\{\left[n_{v} Q\left(\frac{Q}{P}+\frac{\left(n_{v}-1\right)}{n_{v}} T_{v}\right)-\frac{n_{v}{ }^{2} Q^{2}}{2 P}\right]-\left[\frac{T_{v} Q}{n_{v}}\left(1+2+\ldots+\left(n_{v}-1\right)\right)\right]\right\} \\
& =\frac{Q}{2}\left(\left(n_{v}-1\right)\left(1-\frac{n_{v} Q}{T_{v} P}\right)+\frac{n_{v} Q}{T_{v} P}\right),
\end{aligned}
$$

where the cycle time $T_{v}$ is specified as in Figure 1. The vendor's total profit per unit time can now be expressed as 


$$
T P_{v}\left(n_{v}\right)=\frac{c n_{v} Q}{T_{v}}-\frac{A_{v}}{T_{v}}-h_{v} \frac{Q}{2}\left(\left(n_{v}-1\right)\left(1-\frac{n_{v} Q}{T_{v} P}\right)+\frac{n_{v} Q}{T_{v} P}\right) .
$$

Substituting $T_{v}=n_{v} n_{b} T_{d}, T_{d}=q^{1-\beta} /[\alpha(1-\beta)]$, and $Q=n_{b} q$ into expression (6) and simplifying, we obtain the total profit per unit time for the vendor as

$$
T P_{v}\left(n_{v}\right)=c \alpha(1-\beta) q^{\beta}-\frac{\alpha(1-\beta) A_{v}}{n_{v} n_{b} q^{1-\beta}}-h_{v} \frac{n_{b} q}{2}\left(\left(n_{v}-1\right)+\frac{\left(2-n_{v}\right) \alpha(1-\beta) q^{\beta}}{P}\right) .
$$

Taking the first and second derivatives of $T P_{v}\left(n_{v}\right)$ with respect to $n_{v}$ (assumed temporarily to be continuous), we obtain

$$
\begin{aligned}
& \frac{d T P_{v}\left(n_{v}\right)}{d n_{v}}=\frac{\alpha(1-\beta) A_{v}}{n_{b} q^{1-\beta} n_{v}^{2}}-h_{v} \frac{n_{b} q}{2}\left(1-\frac{\alpha(1-\beta) q^{\beta}}{P}\right), \\
& \frac{d^{2} T P_{v}\left(n_{v}\right)}{d n_{v}{ }^{2}}=\frac{-2 \alpha(1-\beta) A_{v}}{n_{b} q^{1-\beta} n_{v}^{3}}<0 .
\end{aligned}
$$

Hence, $T P_{v}\left(n_{v}\right)$ is concave in $n_{v}$. Therefore, the following optimality conditions can be obtained for $n_{v}$

$$
n_{v}\left(n_{v}-1\right) \leq \frac{2 \alpha(1-\beta) A_{v} P q^{\beta}}{h_{v} n_{b}^{2} q^{2}\left(P-\alpha q^{\beta}(1-\beta)\right)} \leq n_{v}\left(n_{v}+1\right)
$$

In the non-coordinated supply chain, the buyer chooses its own optimal policy $\left(q^{*}, n_{b}^{*}\right)$, and the vendor then chooses its optimal number of shipments $n_{v}^{*}$. Thus, total system profit per unit time is obtained as $T P_{N}\left(q^{*}, n_{b}^{*}, n_{v}^{*}\right)=T P_{b}\left(q^{*}, n_{b}^{*}\right)+T P_{v}\left(n_{v}^{*}\right)$.

\section{Coordinated supply chain}

In this section, we consider the situation in which the two parties in the supply chain either cooperate fully or are centrally directed to follow the jointly optimal policy derived by maximizing the total profit $T P_{C}\left(q, n_{b}, n_{v}\right)$. The optimal policy of this coordinated system is found by solving the following problem: 


$$
\begin{aligned}
\underset{q, n_{b}, n_{v}}{\operatorname{Maximize}} & T P_{C}\left(q, n_{b}, n_{v}\right)=\delta \alpha(1-\beta) q^{\beta}-\frac{\alpha(1-\beta)\left[A_{v} /\left(n_{b} n_{v}\right)+A_{b} / n_{b}+S\right]}{q^{1-\beta}} \\
& -\left(\frac{h_{w}\left(n_{b}-1\right)+h_{v} n_{b}\left(n_{v}-1\right)}{2}+\frac{h_{d}(1-\beta)}{2-\beta}\right) q-\frac{h_{v} n_{b}\left(2-n_{v}\right) \alpha(1-\beta) q^{1+\beta}}{2 P} \\
\text { s.t. } & 1 \leq q \leq C_{d} \\
& n_{b}, n_{v} \text { integer. }
\end{aligned}
$$

Taking the first and the second partial derivatives of $\operatorname{TP}\left(q, n_{b}, n_{v}\right)$ with respect to $q$, we obtain

$$
\begin{aligned}
\frac{\partial T P_{C}\left(q, n_{b}, n_{v}\right)}{\partial q}= & \frac{\beta \delta \alpha(1-\beta)}{q^{1-\beta}}+\frac{\alpha(1-\beta)^{2}\left[A_{v} /\left(n_{b} n_{v}\right)+A_{b} / n_{b}+S\right]}{q^{2-\beta}}+\frac{h_{v} n_{b}\left(n_{v}-2\right) \alpha\left(1-\beta^{2}\right) q^{\beta}}{2 P} \\
& -\left(\frac{h_{w}\left(n_{b}-1\right)+h_{v} n_{b}\left(n_{v}-1\right)}{2}+\frac{h_{d}(1-\beta)}{2-\beta}\right) \\
\frac{\partial^{2} T P_{C}\left(q, n_{b}, n_{v}\right)}{\partial q^{2}} & =-\delta \alpha \beta(1-\beta)^{2} q^{\beta-2}-\alpha(1-\beta)^{2}(2-\beta)\left[A_{v} /\left(n_{b} n_{v}\right)+A_{b} / n_{b}+S\right] q^{\beta-3} \\
& -\frac{h_{v} n_{b}\left(2-n_{v}\right) \alpha \beta\left(1-\beta^{2}\right) q^{\beta-1}}{2 P} .
\end{aligned}
$$

It cannot be concluded that $\partial^{2} T P_{C}\left(q, n_{b}, n_{v}\right) / \partial q^{2}$ is necessarily negative. One of two cases may occur depending on the number of shipments $n_{v}$ :

Case 1: $n_{v} \leq 2$

All three terms in $\partial^{2} T P_{C}\left(q, n_{b}, n_{v}\right) / \partial q^{2}$ are negative, and therefore total system profit is concave in $q$ for known values of $n_{b}$ and $n_{v}$. However, there is no closed-form solution for the transfer quantity $q$. We then employ a one-dimensional search algorithm to find its optimal value.

Case 2: $n_{v}>2$

The first two terms in $\partial^{2} T P_{C}\left(q, n_{b}, n_{v}\right) / \partial q^{2}$ are negative. However, the third term is positive. Rewriting $\partial^{2} T P_{C}\left(q, n_{b}, n_{v}\right) / \partial q^{2}$, we have

$$
\frac{\partial^{2} T P_{C}\left(q, n_{b}, n_{v}\right)}{\partial q^{2}}=q^{\beta-3}\left(\vartheta_{3} q^{2}-\vartheta_{1} q-\vartheta_{2}\right)
$$


where $\vartheta_{1}=\delta \alpha \beta(1-\beta)^{2}, \vartheta_{2}=\alpha(1-\beta)^{2}(2-\beta)\left[A_{v} /\left(n_{b} n_{v}\right)+A_{b} / n_{b}+S\right]$, and $\vartheta_{3}=h_{v} n_{b}\left(n_{v}-2\right) \alpha \beta\left(1-\beta^{2}\right) /(2 P)$.

As can be seen directly, $\vartheta_{1}, \vartheta_{2}$, and $\vartheta_{3}$ are all positive. Setting the second partial derivative of $T P_{C}\left(q, n_{b}, n_{v}\right)$ with respect to $q$ equal to zero, and solving, we obtain the two saddle points

$$
\begin{aligned}
& q_{1}=\frac{\vartheta_{1}-\sqrt{\vartheta_{1}^{2}+4 \vartheta_{2} \vartheta_{3}}}{2 \vartheta_{3}}, \\
& q_{2}=\frac{\vartheta_{1}+\sqrt{\vartheta_{1}^{2}+4 \vartheta_{2} \vartheta_{3}}}{2 \vartheta_{3}} .
\end{aligned}
$$

Therefore

$$
\frac{\partial^{2} T P_{C}\left(q, n_{b}, n_{v}\right)}{\partial q^{2}} \begin{cases}<0 & \text { if } q_{1}<q<q_{2}, \\ >0 & \text { otherwise. }\end{cases}
$$

Thus, the total profit function is concave between the two saddle points, and convex when $q \leq q_{1}$ or $q \geq q_{2}$. Moreover, as $\sqrt{\vartheta_{1}^{2}+4 \vartheta_{2} \vartheta_{3}}>\vartheta_{1}$ and $\vartheta_{3}>0$, it follows that $q_{1}<0$ and $q_{2}>0$. Moreover, it can be shown that $\lim _{q \rightarrow 0^{+}} d T P_{C}\left(q, n_{b}, n_{v}\right) / d q=+\infty$ and $\lim _{q \rightarrow+\infty} d T P_{C}\left(q, n_{b}, n_{v}\right) / d q=+\infty$. Therefore, the optimal transfer quantity for any given values of $n_{b}$ and $n_{v}$ is the smallest of the local maximum point, $L M$, and the maximum capacity of the display area, $C_{d}$ (see Figure 2). As there is no closed form solution for the local maximum point, we employ a onedimensional search algorithm to find its value.

\section{Figure 2}

Taking the second partial derivatives of $T P_{C}\left(q, n_{b}, n_{v}\right)$ with respect to $n_{b}$ and $n_{v}$ (relaxing temporarily the integrality requirements), we obtain 


$$
\begin{aligned}
& \frac{\partial^{2} T P_{C}\left(q, n_{b}, n_{v}\right)}{\partial n_{v}^{2}}=-\frac{2 \alpha(1-\beta) A_{v}}{n_{v}^{3} n_{b} q^{1-\beta}}<0, \\
& \frac{\partial^{2} T P_{C}\left(q, n_{b}, n_{v}\right)}{\partial n_{b}^{2}}=-\frac{2 \alpha(1-\beta)\left[A_{b}+A_{v} / n_{v}\right]}{n_{b}^{3} q^{1-\beta}}<0 .
\end{aligned}
$$

Hence, $T P_{C}\left(q, n_{b}, n_{v}\right)$ is concave in $n_{v}$ for given values of $q$ and $n_{b}$, and concave in $n_{b}$ for given values of $q$ and $n_{v}$. Taking the first partial derivative of $T P_{C}\left(q, n_{b}, n_{v}\right)$ with respect to $n_{v}$, and setting it equal to zero, we obtain

$$
n_{v}^{2}=\frac{2 \alpha(1-\beta) A_{v}}{h_{v} n_{b}^{2} q^{2-\beta}\left[1-q^{\beta} \alpha(1-\beta) / P\right]},
$$

which shows that there is a negative relation between $n_{b}$ and the optimal $n_{v}$. Thus, the maximal optimum value of $n_{v}$ for a given $q$ is obtained when $n_{b}=1$.

In order to find the maximal optimum value of $n_{v}$, we also need to find the minimum of $\gamma=q^{2-\beta}\left(1-q^{\beta} \alpha(1-\beta) / P\right)$. Taking the second derivative of $\gamma$ with respect to $q$, we find the saddle point $q^{\prime}$ to be

$$
q^{\prime}=\left(\frac{(2-\beta) P}{2 \alpha}\right)^{1 / \beta}
$$

where $\gamma$ is convex when $q \leq q^{\prime}$, and concave when $q>q^{\prime}$. Taking the first derivative of $\gamma$ with respect to $q$, and equalizing it to zero, we obtain the local minimum and maximum as

$$
\begin{aligned}
& q_{\text {Local min }}=0, \\
& q_{\text {Local max }}=\left(\frac{(2-\beta) P}{2 \alpha(1-\beta)}\right)^{1 / \beta} .
\end{aligned}
$$

Because $P>\alpha C_{d}^{\beta} \geq \alpha q^{\beta}, \gamma$ is a positive function. The minimum of $\gamma$ is obtained at $q=0$ or $q=C_{d}$. However, the minimum transfer quantity is 1 . Therefore, the minimum of $\gamma$ will be

$$
\gamma_{\min }=\min \left\{1-\alpha(1-\beta) / P, C_{d}^{2-\beta}\left(1-C_{d}^{\beta} \alpha(1-\beta) / P\right)\right\} .
$$

Consequently, we have 


$$
n_{v}^{\max }=\left\lceil\sqrt{\frac{2 \alpha(1-\beta) A_{v}}{h_{v} \gamma_{\min }}}\right\rceil .
$$

Similarly, taking the first partial derivative of $T P_{C}\left(q, n_{b}, n_{v}\right)$ with respect to $n_{b}$, and equalizing it to zero, we have

$$
n_{b}^{2}=\frac{2 \alpha(1-\beta)\left(A_{b}+A_{v} / n_{v}\right)}{h_{v} q^{2-\beta}\left[1-q^{\beta} \alpha(1-\beta) / P\right] n_{v}+\left(h_{w}-h_{v}\right) q^{2-\beta}+2 h_{v} \alpha(1-\beta) q^{2} / P} .
$$

As shown above, $\gamma=q^{2-\beta}\left(1-q^{\beta} \alpha(1-\beta) / P\right)$ is positive by assumption. Hence, there is also a negative relation between the optimal $n_{b}$ and any given $n_{v}$. The maximal optimum value of $n_{b}$ is then obtained for $n_{v}=1$. For $n_{v}=1$, the equation above reduces to

$$
n_{b}^{2}\left(n_{v}=1\right)=\frac{2 \alpha(1-\beta)\left(A_{b}+A_{v}\right)}{h_{w} q^{2-\beta}+h_{v} \alpha(1-\beta) q^{2} / P} .
$$

Note, that there is also a negative relation between the optimal $n_{b}$ and any given $q$. The maximal optimum value of $n_{b}$ is then obtained at $q=1$. Consequently, we have

$$
n_{b}{ }^{\max }=\left\lceil\sqrt{\frac{2 \alpha(1-\beta)\left(A_{b}+A_{v}\right)}{h_{w}+h_{v} \alpha(1-\beta) / P}}\right\rceil .
$$

\section{Solution algorithm for joint optimum}

We have specified the following algorithm for determining the jointly optimal values of the three decision variables $n_{v}, n_{b}$, and $q$ in the coordinated model. The algorithm is similar in spirit to the algorithm specified in Section 3.

Step 1. Initialize by setting $n_{v}=1, n_{b}=1$, and $T P_{C}^{o p t}=0$. Compute $n_{v}^{\text {max }}$ and $n_{b}^{\max }$ using Equations (10) and (11).

Step 2. Use a one-dimensional search algorithm (in Case 1 or Case 2 above) to find the (smallest) solution to $\partial \operatorname{TP}_{C}\left(q, n_{b}, n_{v}\right) / \partial q=0$ for $0<q \leq C_{d}$ and the given values of $n_{b}$ and $n_{v}$. 
Step 3. If no solution is found in Step 2, then set $q=C_{d}$. Else, if $q<1$, then set $q=1$ and if $T P_{C}\left(C_{d}, n_{b}, n_{v}\right)>\operatorname{TP}\left(q, n_{b}, n_{v}\right)$, then set $q=C_{d}$.

Step 4. Calculate $\operatorname{TP}_{C}\left(q, n_{b}, n_{v}\right)$ using Equation (9).

Step 5. If $T P_{C}>T P_{C}^{o p t}$, then set $T P_{C}^{o p t}=T P_{C}\left(q, n_{b}, n_{v}\right), n_{v}^{o p t}=n_{v}, n_{b}^{o p t}=n_{b}$, and $q^{o p t}=q$.

Step 6. Increment $n_{b}$ by 1 . If $n_{b} \leq n_{b}^{\max }$, then go to Step 2 .

Step 7. Increment $n_{v}$ by 1 . If $n_{v} \leq n_{v}{ }^{\text {max }}$, then set $n_{b}=1$, and go to Step 2. Otherwise, the current solution is globally optimal.

Obviously, after a finite number of iterations, the algorithm terminates with the optimal solution.

\section{Numerical study}

We first consider a base case with the following data: $\alpha=100, \beta=0.2, P=4500 /$ year, $S=\$ 25 /$ transfer,$\quad A_{b}=\$ 100 /$ order,$\quad A_{v}=\$ 400 /$ setup,$\quad h_{d}=\$ 20 /$ unit $/$ year,$\quad h_{w}=\$ 5 /$ unit $/$ year , $h_{v}=\$ 4 /$ unit/year, $\delta=\$ 30 /$ unit, $c=\$ 20 /$ unit, and $C_{d}=500$. Most of these parameter values have been collected from earlier studies of JELS problems. In order to analyze the effect of stockdependent demand on the benefits of supply chain coordination, we also specify three and twelve levels for the parameters $\alpha$ and $\beta$, respectively. Specifically, $\alpha \in[75,100,125]$, and $\beta \in[0.00,0.05, \ldots, 0.55]$ are used. To represent gains from using coordinated versus noncoordinated policies, we define the percentage gain as $P G=100 \times\left(T P_{C}-T P_{N}\right) / T P_{N}$.

In Table 1, for different values of the demand parameters $\alpha$ and $\beta$, we compare the optimal values of the decision variables in the coordinated and non-coordinated supply chain. The buyer's optimal order quantity $Q=n_{b} q$ is always smaller in the non-coordinated case compared to the coordinated case. However, there are no such general relations between the optimal transfer quantities $q$, or between the vendor's optimal production batches $n_{v} Q$ in the 
coordinated and non-coordinated cases.

Moreover, as $\beta$ increases, the percentage gain $P G$ in the last column of Table 1 grows initially and subsequently declines. This is due to the fact that as demand becomes more sensitive to the stock level in the display area, coordination becomes relatively more profitable. However, because of the limited capacity of the display area, the transfer quantity cannot increase further for values of $\beta$ above a certain level. The decision variables then remain the same. Consequently, the percentage gains start decreasing and eventually vanish.

Another conclusion that can be drawn from Table 1 is that for most of the cases, the benefits of coordination decrease by $\alpha$ for a fixed value of $\beta$. A few exceptions to this general effect are related to the discrete nature of the decision variables $n_{b}$ and $n_{v}$. Thus, as the demand rate $\alpha$ increases, the difference between the coordinated and the non-coordinated supply chain profits generally becomes smaller (assuming no changes in the demand sensitivity).

\section{Table 1}

We also analyze the effect of the unit holding cost in the display area, $h_{d}$, on the benefits of coordination. In order to concentrate on this effect, the capacity of the display area is then assumed not to be binding in the solution, i.e. $C_{d}>M$, where $M$ is a sufficiently large number. From the results in Table 2, we conclude that the percentage gain does not exhibit strong sensitivity with respect to changes in $h_{d}$. Decreasing the unit holding cost to one fourth of its initial value still leaves a percentage gain of more than $13 \%$ from coordination compared to the initial gains of approximately $17 \%$. There is no monotone relationship, however, between the percentage gain and the unit holding cost. The reason appears to be related to the simultaneous discrete changes in the integer multiples $n_{b}$ and $n_{v}$. 


\section{Table 2}

Finally, we examine the effect on the gains of coordination of the unit (net) purchasing price $c$ paid by the buyer to the vendor. For this purpose, we use different levels of the purchasing price $c$ expressed by the ratio $c / \delta$ (for a given value of $\delta$ ). Specifically, we consider $c / \delta \in[-40 \%,-35 \%, \ldots, 0 \%, \ldots, 100 \%]$. However, the purchasing price paid is only an internal transfer from one supply chain member (the buyer) to another (the vendor). Hence, it is not a cost for the supply chain as a whole. Therefore, the total system profit does not change with the unit purchasing (or transfer) price $c$ when the control of the supply chain is coordinated. On the other hand, as $c / \delta$ increases, the buyer's marginal profit from selling the product $\delta-c$ decreases. The optimal transfer quantity from the warehouse to the display area then becomes smaller if the buyer tries to maximize its own profit. Therefore, the number of products in the display area is reduced, and as a result, demand as well as total system profit in the non-coordinated case decreases.

Table 3 shows how the absolute difference $D=T P_{C}-T P_{N}$ and the relative difference $P G$ between total system profits under coordinated and non-coordinated regimes vary by the ratio $c / \delta$. The non-coordinated supply chain's performance is exactly the same as in the coordinated case when the ratio $c / \delta$ is approximately $-25 \%$ (more precisely: $-24.2 \%$ ). In this case, the buyer's optimal order quantity $Q=n_{b} q$ in the non-coordinated supply chain equals the quantity in the coordinated case. Hence, the net unit transfer price $c$ could be employed as a coordination tool in a supply chain dyad with stock-dependent demand. If the two parties set their production, shipment and ordering policies based on a transfer price $c \leq 0$, then the supply chain without further sharing of information performs similarly to a coordinated supply chain. However, coordination of decisions generally also requires a mechanism for sharing of the total profit improvements, as discussed further below. 
In principle, full coordination, if costless, can always be made beneficial for both supply chain parties. For reasons such as costly information or strategic considerations, the parties may not be inclined to share detailed information with each other. Using the precise value of the net unit purchasing price that achieves perfect coordination between the supply chain members is then not the first-best solution either. It requires information sharing about costs and other parameters similarly to what is required in order to obtain the full coordination solution directly. However, because both $P G$ and its sensitivity with respect to variations of the ratio $c / \delta$ are quite low around zero, we suggest that by choosing $c=0$ as the transfer price, it will still be possible to capture most of the potential gains of supply chain coordination in a case such as this with stock-dependent demand. Recall that $c$ is specified as a net unit purchasing price. Therefore, using $c=0$ corresponds to a unit transfer price that equals the vendor's unit acquisition cost. It might be possible to verify this cost with reasonable accuracy and relatively little effort. The advantage of this arrangement is that the buyer and the vendor can obtain some coordination without further costly sharing and verification of information.

Table 3 also shows that the distribution of profit between the vendor and the buyer depends strongly on the ratio $c / \delta$. In particular, the profit for the buyer and for the vendor is negative for large and small unit purchasing prices, respectively. Different values of the transfer price $c$ (normalized as $c / \delta$ ) were used to obtain different solutions and therefore also different total profit values in the non-coordinated case. However, changing the transfer price not only changes the total profit but also transfers profit between the supply chain members. A relatively higher $c$ obviously transfers profit to the vendor and vice versa. In order to obtain final profit levels that are acceptable to both parties and that encourage cooperation, some kind of profit-sharing mechanism needs to be employed as a supplement. For example, a side 
payment might be agreed upon between the buyer and the vendor in order to share the total profits, in particular the gains obtained by a more coordinated solution. Again, however, reaching a conceded profit sharing agreement may require a fair amount of information sharing to motivate the agreement.

A simple way that has been proposed to allocate the joint total profit between the buyer and the vendor is the following (see Ouyang et al., 2004; Wu and Ouyang, 2003; and Goyal, 1976)

$$
\begin{aligned}
& T P_{C v}=\frac{T P_{v}\left(n_{v}\right)}{T P_{N}\left(q, n_{b}, n_{v}\right)} T P_{C}\left(q, n_{b}, n_{v}\right) \\
& T P_{C b}=\frac{T P_{b}\left(q, n_{b}\right)}{T P_{N}\left(q, n_{b}, n_{v}\right)} T P_{C}\left(q, n_{b}, n_{v}\right)
\end{aligned}
$$

where $T P_{C v}$ and $T P_{C b}$ are the resulting final profits for the vendor and the buyer under a coordinated supply chain, respectively. With this arrangement the vendor and the buyer preserve their relative shares of the total profits. However, there are also several other possible arrangements, for example based on game-theoretic approaches.

To conclude this discussion, it should be observed that if $\beta=0$, then $T C_{N}$ and therefore $D$ and $P G$ do not change with $c$. Thus, if there is no relation between the stock in the display area and the consumer demand, then the net unit purchasing price $c$ will have no effect on coordination in the supply-chain dyad. This demonstrates that it is the stock-dependent demand that creates the double marginalization effect discussed above. Spengler (1950) was one of the first to identify the problem of double marginalization; there is coordination failure in a supply chain dyad if each member of the dyad only considers its own net profit margin when making decisions about prices and quantities that affect total profits. Stock-dependent demand and its effect on the buyer's choice of inventory policy correspond to what Jeuland and Shugan (1983) referred to as a 'point-of-sale effort' other than price adjustments in their 
generic analysis of the double marginalization effect for a bilateral monopoly. In the model treated in this paper, the double marginalization effect combines with the coordination problem caused by the production- and inventory-related setup, ordering, transfer, and holding costs also included in the model. In such a case, as shown by Weng (1995), a simple discount scheme is generally not sufficient to coordinate the supply chain dyad. As confirmed above in the numerical analysis with our models, a profit sharing mechanism, such as a side payment mechanism, is then required for coordination of independent parties to be obtained. We refer to Chapters 6-7 in Graves and de Kok (2003) for further discussions about this issue.

\section{Table 3}

\section{Conclusions}

This paper deals with developing an integrated production-inventory model for a twostage supply chain. The contribution of the paper to the joint economic lot-sizing literature is to add the stock-dependency of demand to existing integrated vendor-buyer models. Hence, a more general model is established in which we assume that demand is not constant but sensitive to the amount of inventory displayed on the retailer's shelves. Stock-dependency also incurs a double marginalization effect in a non-coordinated supply chain dyad. Thereby, our study provides a link between the literature on distribution channel coordination and the joint economic lot-sizing literature. The main findings can be summarized as follows.

Taking the relationship between demand and stock into consideration, we find a further advantage of supply chain coordination in the form of an increase in the total selling amount (the demand). When both parties cooperate or are coordinated, the optimal amount of the product in the display area is higher than in the non-coordinated case when the parties 
optimize individually. As a result, end customer demand, and consequently total system profit, is higher for the coordinated supply chain. Assuming that the capacity limitation of the display area is not binding, the numerical results show a strong relation between the improvements obtained with coordination and the stock-sensitivity of demand. This implies that it is particularly beneficial for supply chain parties to cooperate in cases where consumer demand is increased as more of the product is displayed on the retailer's shelves.

The transfer price agreed between the supply chain parties is also found to be a useful tool for partially coordinating the supply chain with only limited sharing of information. This is due to the presence of a double marginalization problem in the supply chain model. The vendor and the buyer can improve the coordination of their production and inventory decision variables by appropriately choosing the transfer price. However, in addition they also need to agree on a suitable profit sharing mechanism in order to allocate the net benefits that can be obtained from the improved coordination. It may require additional information sharing in order to reach such an agreement.

A future research direction might be to extend this study to other product shipment policies, e.g., the geometric shipment policy. It might also be useful to compare coordinated and non-coordinated supply chains under other types of functions for the stock-dependent demand, as well as under stochastic demand settings. In our model we assume that the inventory on the shelf/in the display area is zero before it is replenished. Relaxing this run-out replenishment policy is also an interesting suggestion for future research.

\section{Acknowledgement}

The first two authors' work has been partly supported by grant no. 275-07-0094 from the Danish Social Science Research Council. The authors would like to thank two anonymous 
referees for their valuable comments and helpful suggestions on improving the quality of the paper.

\section{References}

Abbott, H. and Palekar, U.S., 2008. Retail replenishment models with display-space elastic demand. European Journal of Operational Research, 186, 586-607.

Affisco, J.F., Paknejad, M.J., and Nasri, F., 2002. Quality improvement and setup reduction in the joint economic lot size model. European Journal of Operational Research, 142, 497-508.

Balakrishnan, A., Pangburn, M.S., and Stavrulaki, E., 2004. 'Stack them high, let 'em fly': lot sizing policies when inventories stimulate demand. Management Science, 50(5), 630-644.

Baker, R.C. and Urban, T.L., 1988. Single-period inventory dependent demand models. Omega, 16(6), 605-615.

Banerjee, A., 1986. A joint economic-lot-size model for purchaser and vendor. Decision Science, 17, 292-311.

Ben-Daya, M., Darwish, M. and Ertogral, K., 2008. The joint economic lot sizing problem: review and extensions. European Journal of Operational Research, 185, 726-742.

Chan, C.K. and Kingsman, B.G., 2007. Coordination in a single-vendor multi-buyer supply chain by synchronizing delivery and production cycles. Transportation Research Part E, 43, 90-111.

Chang, H.C., Ouyang, L.Y., Wu, K.S., and Ho, C.H., 2006. Integrated vendor-buyer cooperative inventory models with controllable lead time and ordering cost reduction. European Journal of Operational Research, 170, 481-495.

Curşeu, A., van Woensel, T., Fransoo, J., van Donselaar, K., and Broekmeulen, R., 2009. Modelling handling operations in grocery retail stores: an empirical analysis. Journal of the Operational Research Society, 60, 200-214.

Desmet, P. and Renaudin, V., 1998. Estimation of product category sales responsiveness to allocated shelf space. International Journal of Research in Marketing, 15, 443-457.

Ertogral, K., Ben-Daya, M., and Darwish, M., 2007. Production and shipment lot sizing in vendorbuyer supply chain with transportation cost. European Journal of Operational Research, 176, $1592-1606$.

Goyal, S.K., 1976. An integrated inventory model for a single supplier-single customer problem. International Journal of Production Research, 15(1), 107-111.

Goyal, S.K., 1988. A joint economic-lot-size model for purchaser and vendor: A comment. Decision Science, 19, 236-241. 
Goyal, S.K., 1995. A one-vendor multi-buyer integrated inventory model: A comment. European Journal of Operational Research, 82, 209-210.

Goyal, S.K. and Chang, C.T., 2009. Optimal ordering and transfer policy for an inventory with stock dependent demand. European Journal of Operational Research, 196, 177-185.

Graves S.C. and de Kok, A.G., 2003. Handbooks in Operations Research and Management Science: Supply Chain Management: Design, Coordination and Operation, Elsevier Publishing Company, Chapters 6-7.

Gupta, R and Vrat, P., 1986. Inventory model for stock dependent consumption rate. Opsearch, 23, 19-24.

Hariga, M.A., Al-Ahmari, A., and Mohamed A.-R.A., 2007. A joint optimization model for inventory replenishment, product assortment, shelf space and display area allocation decisions. European Journal of Operational Research, 181, 239-251.

Hill, R.M., 1997. The single-vendor single-buyer integrated production-inventory model with a generalized policy. European Journal of Operational Research, 97, 493-499.

Hill, R.M., 1999. The optimal production and shipment policy for the single-vendor single-buyer integrated production-inventory model. International Journal of Production Research, 37, 2463-2475.

Hill, R.M. and Omar, M., 2006. Another look at the single-vendor single-buyer integrated productioninventory problem. International Journal of Production Research, 44(4), 791-800.

Hoque, M. and Goyal, S.K., 2006. A heuristic solution procedure for an integrated inventory system under controllable lead-time with equal or unequal sized batch shipments between a vendor and a buyer. International Journal of Production Economics, 102, 217-225.

Jeuland, A.P. and Shugan, S.M., 1983. Managing channel profits. Marketing Science, 2(3), 239-272.

Khouja, M., 2003. Optimizing inventory decisions in a multi-stage multi-customer supply chain. Transportation Research Part E, 39, 193-208.

Koschat, M.A., 2008. Store inventory can affect demand: empirical evidence from magazine retailing. Journal of Retailing, 84, 165-179.

Lu, L., 1995. A one-vendor multi-buyer integrated inventory model. European Journal of Operational Research, 81, 312-323.

Ouyang, L., Wu, K. and Ho, C., 2004. Integrated vendor-buyer cooperative models with stochastic demand in controllable lead time. International Journal of Production Economics, 92, 255-266. 
Pan, J.C.-H. and Yang, M.-G., 2008. Integrated inventory models with fuzzy annual demand and fuzzy production rate in a supply chain. International Journal of Production Research, 46(3), 753-770.

Sajadieh, M.S., Jokar, M.R.A., and Modarres, M., 2009. Developing a coordinated vendor-buyer model in two-stage supply chains with stochastic lead-times. Computers \& Operations Research, $36,2484-2489$.

Spengler, J.J., 1950. Vertical integration and antitrust policy. The Journal of Political Economy, 58(4), 347-352.

Urban, T.L., 2005. Inventory models with inventory-level-dependent demand: a comprehensive review and unifying theory. European Journal of Operational Research, 162, 792-804.

Wang, Y. and Gerchak, Y., 2001. Supply chain coordination when demand is shelf-space dependent. Manufacturing \& Service Operations Management, 3(1), 82-87.

Warburton, R.D.H., 2009. EOQ extensions exploiting the Lambert $W$ function. European Journal of Industrial Engineering, 3(1), 45-69.

Weng, Z.K., 1995. Channel coordination and quantity discounts. Management Science, 41(9), 1509-1522.

Wu, K. and Ouyang, L., 2003. An integrated single-vendor single-buyer inventory system with shortage derived algebraically. Production Planning \& Control, 14(6), 555-561.

Yang, P.C. and Wee, H.M., 2000. Economic ordering policy of deteriorated item for vendor and buyer: an integrated approach. Production Planning \& Control, 11(5), 474-480.

Zhou, Y.W., Min, J., and Goyal., S.K., 2008. Supply chain coordination under an inventory-leveldependent demand rate. International Journal of Production Economics, 113, 518-527. 


\section{Table Captions}

Table 1. Decision variables of non-coordinated versus coordinated optimization

Table 2. Models' sensitivity to the unit holding cost at the display area

Table 3. Effect of net purchasing unit price on non-coordinated and coordinated optimization

\section{Figure Captions}

Figure 1. Inventory levels at the three stocking points of the supply chain

Figure 2. Total profit function for different values of $q$ if $n_{v}>2$ 


\section{Table 1}

Table 1. Decision variables of non-coordinated versus coordinated optimization

\begin{tabular}{|c|c|c|c|c|c|c|c|c|c|c|c|}
\hline \multicolumn{2}{|c|}{ Parameters } & \multicolumn{4}{|c|}{ Non-coordinated optimization } & \multicolumn{4}{|c|}{ Coordinated optimization } & \multirow{2}{*}{$T P_{C}-T P_{N}$} & \multirow{2}{*}{$P G$} \\
\hline$\alpha$ & $\beta$ & $q$ & $n_{b}$ & $n_{v}$ & $T P_{N}$ & $q$ & $n_{b}$ & $n_{v}$ & $T P_{C}$ & & \\
\hline \multirow[t]{12}{*}{75} & 0.00 & 17.1 & 3 & 2 & 1342.4 & 15.4 & 8 & 1 & 1396.3 & 53.8 & 4.01 \\
\hline & 0.05 & 19.1 & 3 & 2 & 1540.2 & 25.7 & 5 & 1 & 1594.4 & 54.2 & 3.52 \\
\hline & 0.10 & 21.7 & 3 & 2 & 1781.4 & 45.8 & 3 & 1 & 1885.3 & 103.9 & 5.83 \\
\hline & 0.15 & 32.0 & 2 & 2 & 2128.4 & 77.8 & 2 & 1 & 2324.0 & 195.7 & 9.19 \\
\hline & 0.20 & 38.8 & 2 & 2 & 2582.3 & 159.2 & 1 & 1 & 2989.8 & 407.5 & 15.78 \\
\hline & 0.25 & 72.6 & 1 & 3 & 3434.4 & 232.3 & 1 & 1 & 4077.9 & 643.5 & 18.74 \\
\hline & 0.30 & 98.2 & 1 & 2 & 4543.3 & 367.0 & 1 & 1 & 5747.1 & 1203.9 & 26.50 \\
\hline & 0.35 & 143.0 & 1 & 2 & 6265.1 & 500.0 & 1 & 1 & 8389.3 & 2124.2 & 33.91 \\
\hline & 0.40 & 227.8 & 1 & 1 & 9182.4 & 500.0 & 1 & 1 & 11777.5 & 2595.1 & 28.26 \\
\hline & 0.45 & 404.3 & 1 & 1 & 14654.1 & 500.0 & 1 & 1 & 15872.2 & 1218.2 & 8.31 \\
\hline & 0.50 & 500.0 & 1 & 1 & 20755.6 & 500.0 & 1 & 1 & 20755.6 & 0.0 & 0.00 \\
\hline & 0.55 & 500.0 & 1 & 1 & 26477.3 & 500.0 & 1 & 1 & 26477.3 & 0.0 & 0.00 \\
\hline \multirow[t]{12}{*}{100} & 0.00 & 19.7 & 3 & 2 & 1952.0 & 17.7 & 8 & 1 & 2012.6 & 60.6 & 3.10 \\
\hline & 0.05 & 22.4 & 3 & 2 & 2245.3 & 30.4 & 5 & 1 & 2309.4 & 64.1 & 2.86 \\
\hline & 0.10 & 32.7 & 2 & 3 & 2624.4 & 55.8 & 3 & 1 & 2756.9 & 132.5 & 5.05 \\
\hline & 0.15 & 39.4 & 2 & 2 & 3150.6 & 98.1 & 2 & 1 & 3437.8 & 287.2 & 9.11 \\
\hline & 0.20 & 49.3 & 2 & 2 & 3855.1 & 207.1 & 1 & 1 & 4521.9 & 666.8 & 17.30 \\
\hline & 0.25 & 95.2 & 1 & 2 & 5222.6 & 316.2 & 1 & 1 & 6208.1 & 985.5 & 18.87 \\
\hline & 0.30 & 135.1 & 1 & 2 & 7033.3 & 500.0 & 1 & 1 & 8856.9 & 1823.6 & 25.93 \\
\hline & 0.35 & 207.8 & 1 & 2 & 9913.8 & 500.0 & 1 & 1 & 12498.8 & 2585.0 & 26.07 \\
\hline & 0.40 & 351.7 & 1 & 1 & 15111.9 & 500.0 & 1 & 1 & 16953.4 & 1841.5 & 12.19 \\
\hline & 0.45 & 500.0 & 1 & 1 & 22345.7 & 500.0 & 1 & 1 & 22345.7 & 0.0 & 0.00 \\
\hline & 0.50 & 500.0 & 1 & 1 & 28785.3 & 500.0 & 1 & 1 & 28785.3 & 0.0 & 0.00 \\
\hline & 0.55 & 500.0 & 1 & 1 & 36337.6 & 500.0 & 1 & 1 & 36337.6 & 0.0 & 0.00 \\
\hline \multirow[t]{12}{*}{125} & 0.00 & 22.0 & 3 & 2 & 2578.3 & 19.8 & 8 & 1 & 2644.3 & 66.0 & 2.56 \\
\hline & 0.05 & 25.4 & 3 & 2 & 2974.0 & 40.8 & 4 & 1 & 3048.9 & 74.9 & 2.52 \\
\hline & 0.10 & 37.7 & 2 & 2 & 3498.8 & 65.4 & 3 & 1 & 3664.3 & 165.5 & 4.73 \\
\hline & 0.15 & 46.4 & 2 & 2 & 4228.0 & 118.2 & 2 & 1 & 4611.2 & 383.1 & 9.06 \\
\hline & 0.20 & 86.6 & 1 & 3 & 5487.3 & 255.9 & 1 & 1 & 6162.6 & 675.3 & 12.31 \\
\hline & 0.25 & 118.8 & 1 & 2 & 7182.3 & 404.6 & 1 & 1 & 8524.7 & 1342.4 & 18.69 \\
\hline & 0.30 & 175.1 & 1 & 2 & 9806.1 & 500.0 & 1 & 1 & 12100.5 & 2294.4 & 23.40 \\
\hline & 0.35 & 281.2 & 1 & 1 & 14162.8 & 500.0 & 1 & 1 & 16608.4 & 2445.6 & 17.27 \\
\hline & 0.40 & 497.6 & 1 & 1 & 22093.8 & 500.0 & 1 & 1 & 22129.2 & 35.5 & 0.16 \\
\hline & 0.45 & 500.0 & 1 & 1 & 28819.3 & 500.0 & 1 & 1 & 28819.3 & 0.0 & 0.00 \\
\hline & 0.50 & 500.0 & 1 & 1 & 36815.0 & 500.0 & 1 & 1 & 36815.0 & 0.0 & 0.00 \\
\hline & 0.55 & 500.0 & 1 & 2 & 46265.6 & 500.0 & 1 & 2 & 46265.6 & 0.0 & 0.00 \\
\hline
\end{tabular}




\section{Table 2}

Table 2. Models' sensitivity to the unit holding cost at the display area

\begin{tabular}{c|cccccc|ccccccc|c}
\hline \multirow{2}{*}{$h_{d}$} & \multicolumn{5}{|c|}{ Non-coordinated optimization } & \multicolumn{5}{c|}{ Coordinated optimization } & $P G$ \\
\cline { 2 - 10 } & $q$ & $n_{b}$ & $n_{v}$ & $T P_{b}$ & $T P_{v}$ & $T P_{N}$ & $q$ & $n_{b}$ & $n_{v}$ & $T P_{C b}$ & $T P_{C v}$ & $T P_{C}$ & $(\%)$ \\
\hline 20 & 49.28 & 2 & 2 & 917.6 & 2937.5 & 3855.1 & 207.05 & 1 & 1 & 1076.3 & 3445.6 & 4521.9 & 17.30 \\
17 & 82.25 & 1 & 2 & 1017.4 & 3230.5 & 4247.9 & 241.44 & 1 & 1 & 1154.3 & 3665.3 & 4819.6 & 13.46 \\
14 & 97.26 & 1 & 2 & 1136.4 & 3391.2 & 4527.6 & 291.42 & 1 & 1 & 1298.3 & 3874.3 & 5172.6 & 14.25 \\
11 & 120.72 & 1 & 2 & 1280.4 & 3586.1 & 4866.5 & 370.46 & 1 & 1 & 1475.8 & 4133.6 & 5609.4 & 15.27 \\
8 & 162.87 & 1 & 1 & 1466.3 & 3870.8 & 5337.1 & 513.01 & 1 & 1 & 1700.0 & 4487.8 & 6187.8 & 15.94 \\
5 & 261.09 & 1 & 1 & 1738.0 & 4468.1 & 6206.1 & 837.53 & 1 & 1 & 1975.2 & 5078.2 & 7053.4 & 13.65
\end{tabular}




\section{Table 3}

Table 3. Effect of net purchasing unit price on non-coordinated and coordinated optimization

\begin{tabular}{cccc}
\multicolumn{4}{c}{ Coordinated optimization } \\
\hline$q$ & $n_{b}$ & $n_{v}$ & $T P_{C}$ \\
\hline 207.05 & 1 & 1 & 4521.9 \\
\hline
\end{tabular}

\begin{tabular}{|c|c|c|c|c|c|c|c|c|c|}
\hline \multicolumn{2}{|c|}{ Parameters } & \multicolumn{6}{|c|}{ Non-coordinated optimization } & \multirow{2}{*}{$T P_{C}-T P_{N}$} & \multirow{2}{*}{$P G$} \\
\hline$\delta$ & $c / \delta$ & $q$ & $n_{b}$ & $n_{v}$ & $T P_{b}$ & $T P_{v}$ & $T P_{N}$ & & \\
\hline \multirow[t]{29}{*}{30} & $-40 \%$ & 237.01 & 1 & 1 & 7797.1 & -3293.8 & 4503.3 & 18.6 & 0.41 \\
\hline & $-35 \%$ & 227.56 & 1 & 1 & 7440.3 & -2927.4 & 4512.9 & 9.0 & 0.20 \\
\hline & $-30 \%$ & 218.23 & 1 & 1 & 7086.5 & -2567.4 & 4519.1 & 2.8 & 0.06 \\
\hline & $-25 \%$ & 209.03 & 1 & 1 & 6735.7 & -2213.9 & 4521.8 & 0.1 & 0.00 \\
\hline & $-20 \%$ & 199.95 & 1 & 1 & 6387.9 & -1867.2 & 4520.7 & 1.2 & 0.03 \\
\hline & $-15 \%$ & 191.01 & 1 & 1 & 6043.2 & -1527.6 & 4515.6 & 6.3 & 0.14 \\
\hline & $-10 \%$ & 182.22 & 1 & 1 & 5701.8 & -1195.4 & 4506.3 & 15.6 & 0.35 \\
\hline & $-5 \%$ & 173.57 & 1 & 1 & 5363.5 & -871.0 & 4492.6 & 29.3 & 0.65 \\
\hline & $0 \%$ & 165.08 & 1 & 1 & 5028.6 & -554.6 & 4474.1 & 47.8 & 1.07 \\
\hline & $5 \%$ & 156.76 & 1 & 1 & 4697.1 & -246.5 & 4450.6 & 71.3 & 1.60 \\
\hline & $10 \%$ & 148.61 & 1 & 2 & 4369.1 & 62.6 & 4431.7 & 90.2 & 2.04 \\
\hline & $15 \%$ & 140.64 & 1 & 2 & 4044.6 & 380.9 & 4425.5 & 96.4 & 2.18 \\
\hline & $20 \%$ & 132.87 & 1 & 2 & 3723.7 & 690.3 & 4414.0 & 107.9 & 2.44 \\
\hline & $25 \%$ & 125.30 & 1 & 2 & 3406.5 & 990.5 & 4397.0 & 124.9 & 2.84 \\
\hline & $30 \%$ & 117.95 & 1 & 2 & 3093.1 & 1281.2 & 4374.3 & 147.6 & 3.38 \\
\hline & $35 \%$ & 110.82 & 1 & 2 & 2783.5 & 1562.0 & 4345.4 & 176.5 & 4.06 \\
\hline & $40 \%$ & 103.94 & 1 & 2 & 2477.7 & 1832.5 & 4310.3 & 211.6 & 4.91 \\
\hline & $45 \%$ & 97.30 & 1 & 2 & 2176.0 & 2092.6 & 4268.6 & 253.3 & 5.93 \\
\hline & $50 \%$ & 90.94 & 1 & 2 & 1878.2 & 2342.0 & 4220.2 & 301.7 & 7.15 \\
\hline & $55 \%$ & 84.85 & 1 & 2 & 1584.5 & 2580.4 & 4164.9 & 357.0 & 8.57 \\
\hline & $60 \%$ & 79.05 & 1 & 3 & 1294.8 & 2818.1 & 4113.0 & 408.9 & 9.94 \\
\hline & $65 \%$ & 73.55 & 1 & 3 & 1009.3 & 3054.4 & 4063.7 & 458.2 & 11.27 \\
\hline & $70 \%$ & 46.73 & 2 & 2 & 744.1 & 3068.2 & 3812.2 & 709.7 & 18.62 \\
\hline & $75 \%$ & 43.12 & 2 & 2 & 487.3 & 3254.9 & 3742.2 & 779.7 & 20.84 \\
\hline & $80 \%$ & 39.75 & 2 & 2 & 234.6 & 3430.9 & 3665.5 & 856.4 & 23.36 \\
\hline & $85 \%$ & 28.54 & 3 & 2 & -12.3 & 3451.2 & 3438.9 & 1083.0 & 31.49 \\
\hline & $90 \%$ & 26.30 & 3 & 2 & -245.0 & 3606.0 & 3361.1 & 1160.8 & 34.54 \\
\hline & $95 \%$ & 20.26 & 4 & 2 & -473.4 & 3639.3 & 3165.9 & 1356.0 & 42.83 \\
\hline & $100 \%$ & 18.73 & 4 & 2 & -690.7 & 3778.9 & 3088.2 & 1433.7 & 46.43 \\
\hline
\end{tabular}




\section{Figure 1}

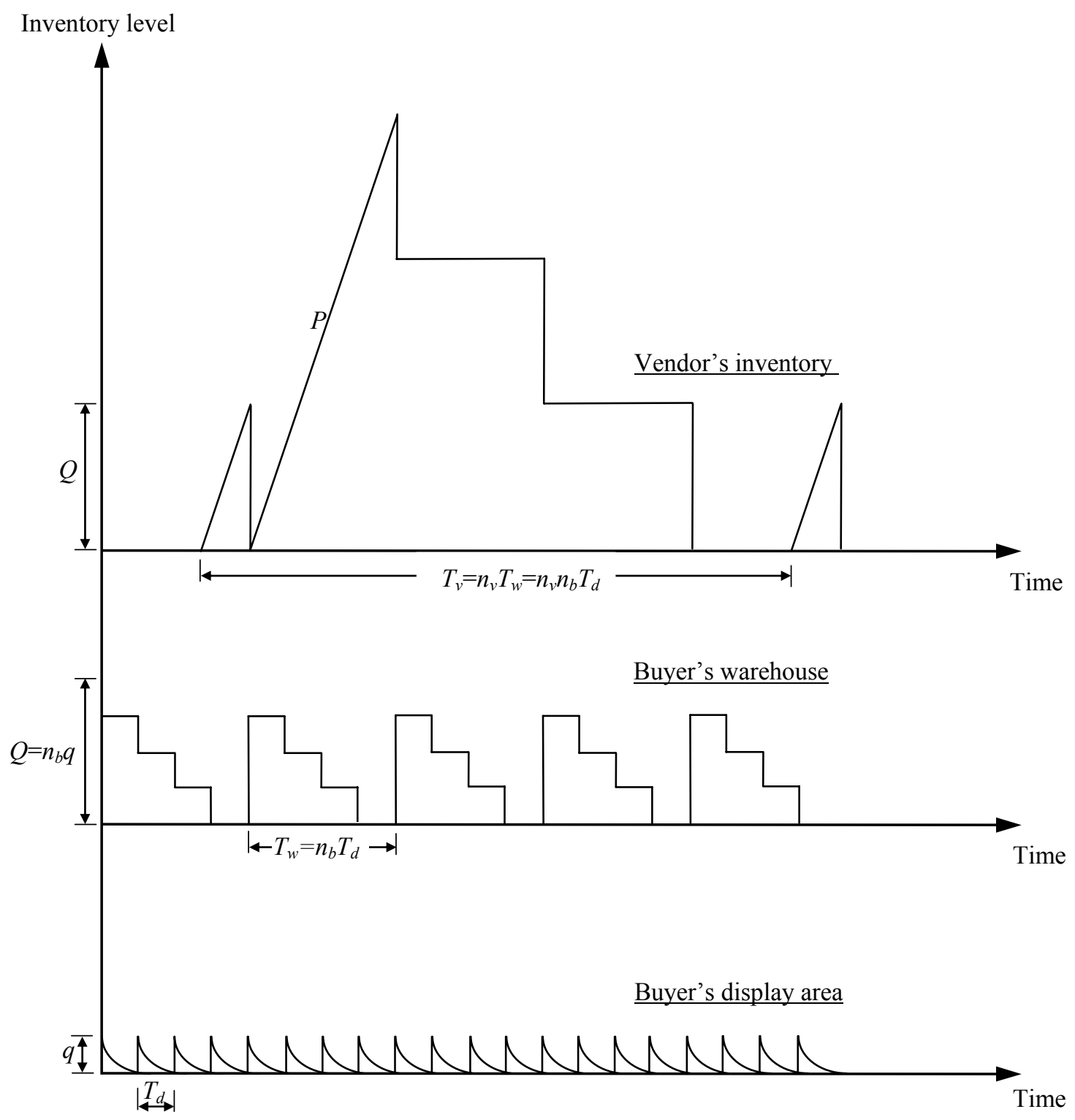

Figure 1. Inventory levels at the three stocking points of the supply chain 
Figure 2
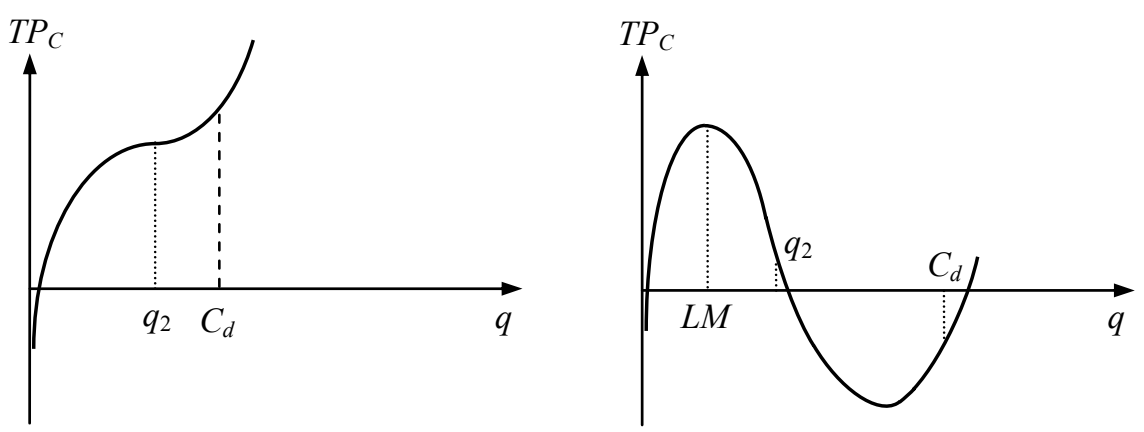

Figure 2. Total profit as a function of $q$ for $n_{v}>2$ 